\title{
Microscopic Analysis of High Lithium-Ion Conducting Glass-Ceramic Sulfides
}

\author{
Mansoo Park*, Wo Dum Jung*, Sungjun Choi*, Kihyun Son*, Hun-Gi Jung**, Byung-Kook Kim*, \\ Hae-Weon Lee*, Jong-Ho Lee ${ }^{*, \dagger}$, and Hyoungchul Kim** \\ *High-Temperature Energy Materials Research Center, Korea Institute of Science and Technology, Seoul 02792, Korea \\ ${ }^{* *}$ Center for Energy Convergence, Korea Institute of Science and Technology, Seoul 02792, Korea
}

(Received July 22, 2016; Revised August 23, August 24, 2016; Accepted August 25, 2016)

\begin{abstract}
We explore the crystalline structure and phase transition of lithium thiophosphate $\left(\mathrm{Li}_{7} \mathrm{P}_{3} \mathrm{~S}_{11}\right)$ solid electrolyte using electron microscopy and X-ray diffraction. The glass-like $\mathrm{Li}_{7} \mathrm{P}_{3} \mathrm{~S}_{11}$ powder is prepared by the high-energy mechanical milling process. According to the energy dispersive X-ray spectroscopy (EDS) and selected area diffraction (SAD) analysis, the glass powder shows chemical homogeneity without noticeable contrast variation at any specific spot in the specimen and amorphous SAD ring patterns. Upon heating up to $260^{\circ} \mathrm{C}$ the glass $\mathrm{Li}_{7} \mathrm{P}_{3} \mathrm{~S}_{11}$ powder becomes crystallized, clearly representing crystal plane diffraction contrast in the high-resolution transmission electron microscopy image. We further confirm that each diffraction spot precisely corresponds to the diffraction from a particular $\mathrm{Li}_{7} \mathrm{P}_{3} \mathrm{~S}_{11}$ crystallographic structure, which is also in good agreement with the previous X-ray diffraction results. We expect that the microscopic analysis with EDS and SAD patterns would permit a new approach to study in the atomic scale of other lithium ion conducting sulfides.
\end{abstract}

Key words : Sulfide lithium ion conductor, Microscopic analysis, Phase transition, Transmission electron microscopy, All-solid-state battery

\section{Introduction}

$\mathbf{A}^{11}$ ll-solid-state battery highlighted as the future rechargeable battery technology in such application areas as an electric vehicle and an energy storage system refers to a battery where all battery components are composed of solidstate elements. The all-solid-state batteries dramatically supplement poor safety and durability as inherent disadvantages of the existing rechargeable batteries containing a liquid electrolyte through utilization of solid electrolytes, and have the features of being able to simultaneously realize high voltage and high energy density for the cell. ${ }^{1-4)}$

As mentioned earlier, the development of high performance with solid electrolytes may be considered as one of the most core technology features for the all-solid-state batteries where conversion to solid phases is realized for all components including solidified electrolyte. Considering the commercialized level of actual lithium rechargeable batteries based on a liquid electrolyte, the minimum value required for ionic conductivity of solid electrolyte is known to be on the level of about $1 \mathrm{mS} / \mathrm{cm}$ at room temperature. ${ }^{3)}$

\footnotetext{
${ }^{\dagger}$ Corresponding author: Jong-Ho Lee

E-mail : jongho@kist.re.kr

Tel : +82-2-958-5532 Fax : +82-2-958-5529

${ }^{+}$Corresponding author: Hyoungchul Kim

E-mail : hyoungchul@kist.re.kr

Tel : +82-2-958-5519 Fax : +82-2-958-5529
}

In the last 30 years, extensive studies on solid electrolyte materials have been globally implemented to develop a high ionic conductivity for such solid electrolytes. ${ }^{3-11)}$ In this process, sulfide-based solid electrolytes are exhibiting the performance which has most closely approached the commercialization stage among all inorganic-based materials with advantages of excellent lithium ionic conductivity and roomtemperature formability based on the material ductility. ${ }^{3,5)}$

Although the amorphous system most widely used among sulfide-based solid electrolytes is stable at room temperature with an advantage that its performance degradation is small, it suffers from the structural difficulty for dramatic improvement of ionic conductivity beyond the level discovered thus far. In general, a method of increasing concentrations of the conduction carrier for glass, i.e. lithium, has been frequently utilized as a means to improve the ionicconductivity of glass matrix, and many studies for increasing the concentration of lithium ions have been conducted by combining several types of oxides such as $\mathrm{Li}_{2} \mathrm{O}$, etc. ${ }^{3,5,7)}$ Meanwhile, the amorphous and partly crystalline sulfide ceramics materials which were first proposed more than 10 years ago have been much studied as an ionic conductor exhibiting the higher lithium ionic conduction characteristics than fully amorphous materials. ${ }^{3,5,8,9)}$ Such sulfide materials form glass or glass-ceramics depending on the mixing ratios between $\mathrm{Li}_{2} \mathrm{~S}$ and $\mathrm{P}_{2} \mathrm{~S}_{5}$, mechanical alloying and heat treatment conditions, ${ }^{3,5,8)}$ and are generally known to show the highest lithium ionic conductivity when $\mathrm{P}_{2} \mathrm{~S}_{5}$ has a mole 
ratio of about $30 \%$ although it varies with the extent of crystallization to the highly conductive phase.9) Particularly, $\mathrm{Li}_{10} \mathrm{GeP}_{2} \mathrm{~S}_{12}$ with a $3 \mathrm{D}$ framework for conducting structure has been recently reported as having a conductivity on the equivalent level to that of organic liquid electrolytes, i.e. a high ionic conductivity of about $12 \mathrm{mS} / \mathrm{cm} .{ }^{10)}$

However, crystallographic understanding and direct observation through electron microscope of the sulfide solid electrolytes for which diversified studies have been conducted for a long time still remain at an extremely elementary level considering the high attention paid to the material. The representative reasons may be summarized as; i) a high water reactivity exhibited by sulfide-based materials, ii) vulnerability to electron and ion beams, iii) highly volatile characteristics of constituent elements of the material, iv) a high temperature dependence of the crystal structures, v) complex structures of crystal and amorphous phases, etc. ${ }^{3,11,12)}$ Such inherent limitations of the material act as fatal constraints for the analysis by electron microscope, and particularly transmission electron microscope (TEM). ${ }^{12)}$ For crystallographic analysis in the articles reported on sulfide-based solid electrolyte materials thus far, X-ray diffraction (XRD) technique based on powders was used primarily, ${ }^{9,10,11)}$ and only limited TEM studies (shape image and composition distribution) confined to wide area low-resolution have been realized. ${ }^{3,12-14)}$ In particular, information on the crystal structures of glass-ceramic obtained from bulk analysis such as XRD failed in actual cross verification and confirmation of the definitive distribution form of the highly conductive phase through advanced microscopic analysis, which was one of the major limitations appearing thus far in the studies on sulfide-based solid electrolytes.

In this study, phase transition phenomenon of Li-ion conducting sulfide solid electrolyte based on Li-P-S (to be referred to as LPS hereafter) was observed on the basis of TEM as a high-resolution microscopic analysis technique, and a study for affirming crystallographic uniqueness of the glass-ceramic structure was conducted by using the selected area diffraction (SAD) patterns and high-magnification bright-field images. In particular, an emphasis was placed on overcoming limitations in electron microscope analysis of sulfides vulnerable to electron beam and ion beam, and on crystallographic understanding of the material and process limitations through actual observation of microstructures. Through such in-depth crystallographic understanding expanded to microscopic area, a theoretical and technical foundation required for the future development of improved Li-ion conducting sulfide solid electrolytes will be provided in the present article.

\section{Experimental Procedure}

Li-ion conducting LPS-based sulfide solid electrolyte was prepared by employing a dry mechanical alloying method using a planetary mill (PM 200, Retsch) equipment and high temperature heat treatment technique. Raw material powders of $\mathrm{Li}_{2} \mathrm{~S}$ (Aldrich) of $\mathrm{P}_{2} \mathrm{~S}_{5}$ (Aldrich) having a total weight of $2 \mathrm{~g}$ were mixed to the molar ratio of $7: 3$ and filled a $\mathrm{ZrO}_{2}$ container of $50 \mathrm{~mL}$ capacity together with $\mathrm{ZrO}_{2}$ balls of $5 \mathrm{~mm}$ in diameter (the ball to powder ratio $=30$ ), followed by sealing. In a similar way to the experimental conditions reported in the existing literature, the planetary mill involved high energy mixing-pulverization for $8 \mathrm{~h}$ under the condition of $650 \mathrm{rpm}$, the glass powder obtained through which was placed in a borosilicate glass container filled with argon gas and sealed, and was subjected to high-temperature heat treatment at $260^{\circ} \mathrm{C}$ for $2 \mathrm{~h}$, resulting in synthesis of LPS-based solid electrolyte with a glass-ceramic crystal structure. ${ }^{3,5,8,9)}$ All powder preparation processes excluding planetary milling which used a sealed container as well as high-temperature heat treatment were executed in a glove box which maintained the level of water and oxygen to be less than $0.1 \mathrm{ppm}$ so as to suppress any degradation of samples due to a change in external conditions which could perhaps occur.

Crystallographic changes in thus-obtained glass and glass-ceramic samples were checked by XRD (D/Max 2500, Rigaku) analysis. XRD analysis was performed by placing the powder sample in a sealed measuring container to shut off any sample contamination, and by using $\mathrm{Cu} \mathrm{Ka}$ light source under the conditions of $10 \sim 60^{\circ}$ for scanning range, $2 \%$ min for scanning speed, and $0.026^{\circ}$ for step size. Electrical conductivity of the synthesized samples was measured by electrochemical impedance spectroscopy (EIS) using a frequency response analyzer (1260, Solartron) and an electrochemistry interface (1287, Solartron) equipment after loading the powder in a metal mold for subsequent forming with uniaxial pressurization to the pressure of $300 \mathrm{MPa}$. Alternating current impedance experiment was conducted at room temperature under the conditions of $10 \sim 10^{7} \mathrm{~Hz}$ for frequency and $50 \mathrm{mV}$ for voltage, and an equivalent impedance model was composed on the basis of the obtained data, from which resistance values were obtained and calculations were made by reflecting a shape factor for the measurement sample. For the measurement of electronic conductivity $\left(\sigma_{e}\right)$, Hebb-Wagner direct current polarization method $^{15)}$ was employed. The potential relaxation behavior observed by applying $60 \mathrm{nA}$ of DC current to a Li/electrolyte/SUS half-cell was checked, and the electronic conductivity was calculated according to the following relational expression of $\sigma_{e}=-\left(i_{e} / U\right)(l / A)$, where $i_{e}$ is the applied DC current, $U$ is the changed amount of potential, $l$ is the specimen thickness, $A$ is the cross section area of specimen. To check for the electrochemical stability of solid electrolyte samples, measurement of cyclic voltammetry (CV) curves was also executed by composing a Li/electrolyte/SUS half-cell under the conditions of $1 \mathrm{mV}$ per sec for scanning speed in the range of -0.5 to $5 \mathrm{~V}$ using the same equipment as that for EIS.

To obtain high-resolution bright-field images and SAD patterns, TEM equipment (Tecnai F20, FEI) with a field 
emission gun mounted was employed. Also, for fast and accurate image and quantitative analysis of constituent element distribution in wide area/ local region, a high-speed energy dispersive X-ray spectroscopy (EDS) with 4 detectors of silicon-drift type mounted and a TEM (Talos F200X, FEI) were employed together. TEM specimens were prepared by scattering electrolyte powder onto a copper TEM grid coated with carbon film. Also, by considering the specimen characteristics vulnerable to atmosphere, a vacuum transfer holder was utilized to minimize air exposure upon TEM loading of the specimen.

\section{Results and Discussion}

When the raw materials of $\mathrm{Li}_{2} \mathrm{~S}$ and $\mathrm{P}_{2} \mathrm{~S}_{5}$ powders are mixed to the molar ratio of $7: 3$ and high-energy milling is conducted, a glass structure is formed by $\mathrm{P}_{2} \mathrm{~S}_{5}$ acting as a glass former, and $\mathrm{Li}_{2} \mathrm{~S}$ as a network modifier. Such LPS $(7: 3)$ glass evolves into a glass-ceramic structure with a high $\mathrm{Li}$ ion conducting phase through heat treatment above about $240^{\circ} \mathrm{C}$ for crystalization. ${ }^{9)}$ Thus-produced crystalline structure is known to be $\mathrm{Li}_{7} \mathrm{P}_{3} \mathrm{~S}_{11}$, ${ }^{9,16)}$ The chemical reaction formula related to this process is shown as $7 \mathrm{Li}_{2} \mathrm{~S}+3 \mathrm{P}_{2} \mathrm{~S}_{5} \rightarrow$ $2 \mathrm{Li}_{7} \mathrm{P}_{3} \mathrm{~S}_{11}$.

Figure 1 shows XRD patterns before and after heat treatment of the LPS $(7: 3)$ material. In the same manner as mentioned in the prior literature, ${ }^{9,16)}$ generation of considerably broad peaks instead of sharp peaks can be seen in all regions, considering the XRD patterns before heat treatment, i.e. after milling. In particular, the peak near $30^{\circ}$ as the main peak for $\mathrm{Li}_{7} \mathrm{P}_{3} \mathrm{~S}_{11}$ shown together with the reference is affirmed to be observed as a broad peak in the XRD

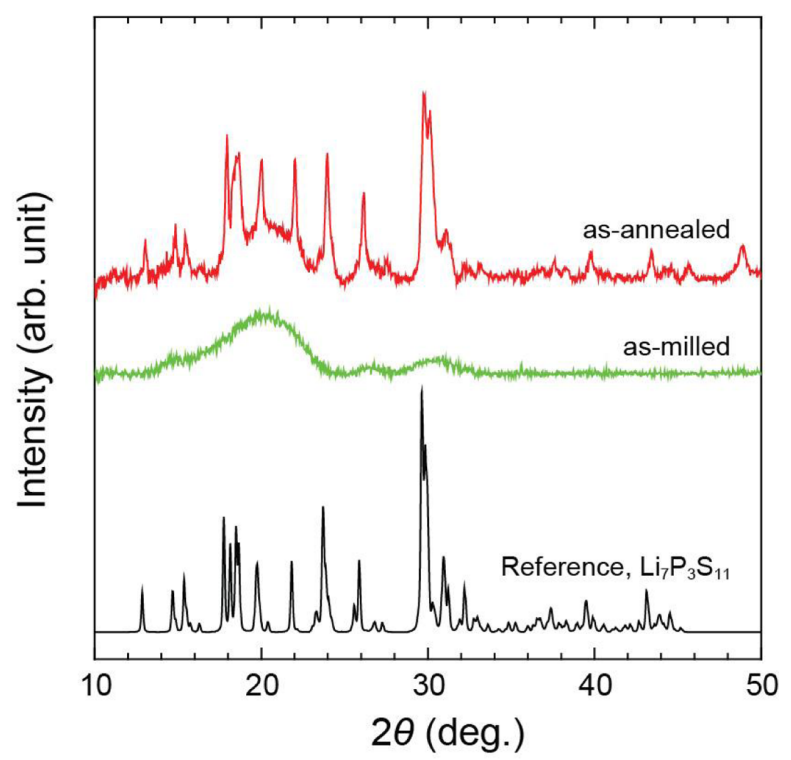

Fig. 1. XRD patterns of an as-milled (green) and annealed (red) LPS (7:3) powder, showing the glass and high lithium-ion conducting crystalline phase. The reference XRD pattern (black) of $\operatorname{Li}_{7} \mathrm{P}_{3} \mathrm{~S}_{11}$ crystal is also shown. spectrum before heat treatment. Through this observation, although phase formation of $\mathrm{Li}_{7} \mathrm{P}_{3} \mathrm{~S}_{11}$ occurs properly in the sample prior to heat treatment, the phase is shown to be amorphous due to the milling energy. Considering the XRD pattern for the LPS $(7: 3)$ heat treated at $260^{\circ} \mathrm{C}$ for $2 \mathrm{~h}$, the formation of the $\mathrm{Li}_{7} \mathrm{P}_{3} \mathrm{~S}_{11}$ phase is confirmed by the close agreement with the reference diffraction peaks of $\mathrm{Li}_{7} \mathrm{P}_{3} \mathrm{~S}_{11}$. As a reference, the wide and broad peak observed near $20^{\circ}$ before and after heat treatment was due to the diffraction of the film used in order to prevent atmospheric exposure. In addition, ionic conductivities of the LPS (7:3) sample before and after heat treatment measured through EIS analysis are 0.4 and $2.0 \mathrm{mS} / \mathrm{cm}$, respectively (electronic conductivity being about $9.7 \mathrm{mS} / \mathrm{cm}$ ), which correspond to those in the literature, ${ }^{9)}$ verifying that there is no problem with the material synthesis process in the present article. Furthermore, electrochemical stability behavior of the LPS (7:3) solid electrolyte was also checked through the CV measurement, as shown in Fig. 2. Since no specific decomposition was observed up to $5 \mathrm{~V}$ band as reported in the literature, ${ }^{17)}$ retaining of a wide electrochemical window is thereby confirmed.

For the LPS (7:3) sample which is affirmed to have a glass structure based on the XRD analysis, TEM analysis was conducted. As mentioned in the introduction, the sulfide-based samples involve several inherent difficulties in performing TEM analysis. In particular, the atmospheric exposure problem and vulnerability to electron and ion beams impose limitations to the sample preparation for TEM, high-resolution TEM image and quantitative element analysis by EDS. Fig. 3 shows a bright-field image and a SAD pattern for the LPS $(7: 3)$ sample before heat treatment. A broad diffraction ring of ( $2 \overline{1} \overline{1})$ as the main electron diffraction peak for $\mathrm{Li}_{7} \mathrm{P}_{3} \mathrm{~S}_{11}$ appears in the $\mathrm{SAD}$ pattern result, which is in agreement with the XRD analysis described earlier. The wider and lighter diffraction ring

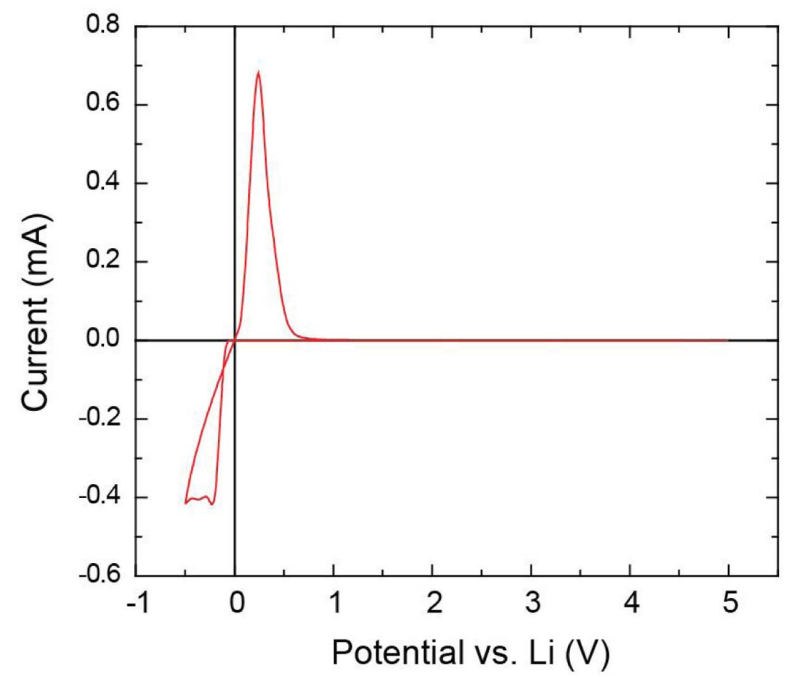

Fig. 2. Cyclic voltammogram for a $\mathrm{Li}_{2} / \mathrm{Li}_{7} \mathrm{P}_{3} \mathrm{~S}_{11} / \mathrm{SUS}$ cell at the first cycle. 


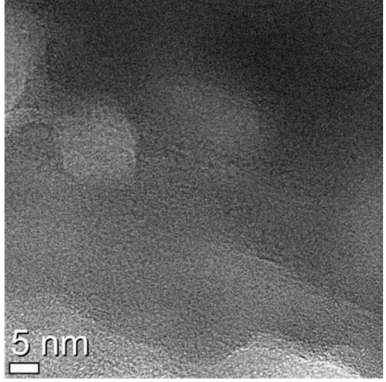

(a)

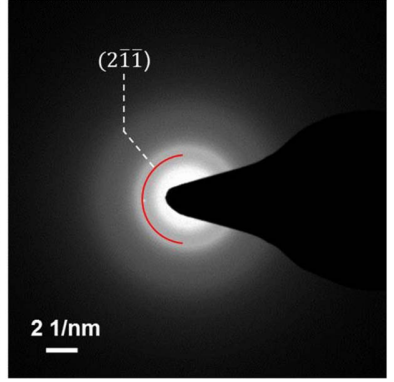

(b)
Fig. 3. (a) Bright field TEM image and (b) SAD pattern of an as-milled LPS (7:3) particle with glass structure.

than for the nanocrystalline diffraction pattern indicates that LPS $(7: 3)$ before heat treatment has an amorphous structure. In addition, considering the high-magnification TEM image of Fig. 3(a), diffractions of lattice planes shown in the crystalline structure does not appear which is in agreement with amorphous results of XRD and SAD. As a reference, the $\mathrm{SAD}$ pattern with the interplanar distance being closer than ( $2 \overline{1} \overline{1})$ was not quite visible due to obscuring by the bright electron transmission beam.

Through high-speed EDS mapping technique with 4 detectors of silicon drift type mounted, element distribution and quantitative analysis was conducted. Fig. 4 shows elemental distributions for $\mathrm{P}$ and $\mathrm{S}$ within the LPS $(7: 3)$ sample before heat treatment; $\mathrm{Li}$ is, unfortunately, not detected due to itslightweight in EDS analysis. Considering the atomic distributions of $\mathrm{P}$ and $\mathrm{S}$ shown in Fig. 4, each element is uniformly distributed with no variations in contrast throughout the whole region of the sample. This suggests that the raw material powders of $\mathrm{Li}_{2} \mathrm{~S}$ and $\mathrm{P}_{2} \mathrm{~S}_{5}$ put in before
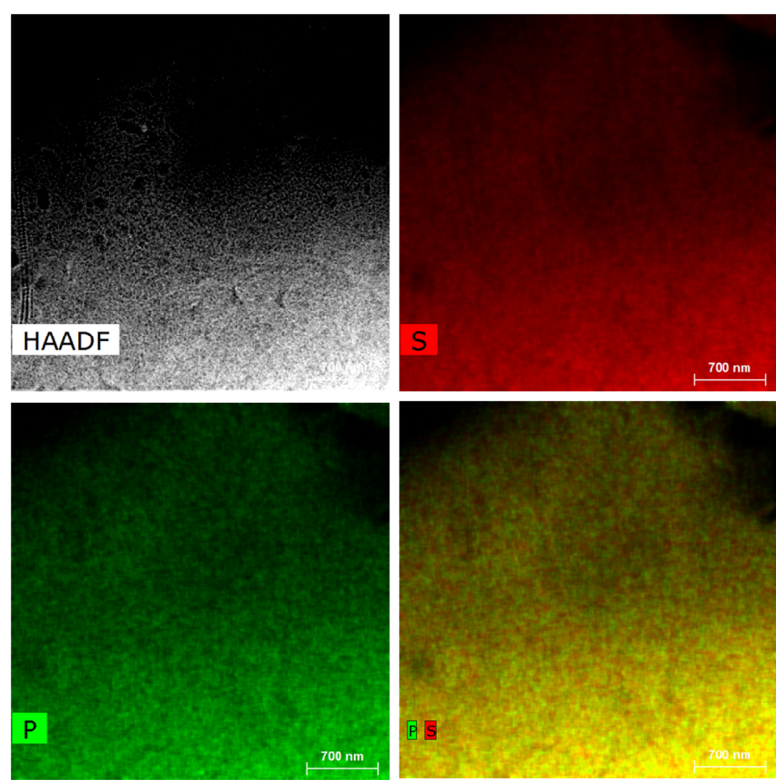

Fig. 4. TEM and EDS elemental mapping images of asmilled LPS $(7: 3)$ particle. The green and red areas correspond to the elements of $\mathrm{P}$ and $\mathrm{S}$, respectively. milling are chemically mixed for satisfactory formation of the glass of $\mathrm{Li}_{7} \mathrm{P}_{3} \mathrm{~S}_{11}$. The composition of the amorphous matrix confirmed through EDS was measured to be about 78 and 22 at.\% for S and P, respectively; Li was excluded and an accurate quantitative analysis was impossible. However, the formation of $\mathrm{Li}_{7} \mathrm{P}_{3} \mathrm{~S}_{11}$ glass with an accurate composition could be confirmed based on the fact that the ratio measured between $\mathrm{P}$ and $\mathrm{S}$ is almost $3: 11$.

For the crystalline sample of the LPS $(7: 3)$ with heat treatment applied, the same analysis was also performed using TEM, aiming at actual observation of $\mathrm{Li}_{7} \mathrm{P}_{3} \mathrm{~S}_{11}$ microstructure as well as broad crystallographic understanding expanded to micro areas. Fig. 5 shows high-magnification bright-field image and SAD pattern of the LPS $(7: 3)$ glassceramic powder after heat treatment at $260^{\circ} \mathrm{C}$ for $2 \mathrm{~h}$. Since sulfide materials are vulnerable to electron beam so that high-magnification bright-field images are very difficult to obtain, the difficulty in analyzing such micro areas is precisely one of the most major limitations for microscopic analysis studies on sulfide-based solid electrolytes. Nevertheless, it was possible to obtain high-magnification brightfield images after several attempts of trial and error were made, and the crystal plane frequently observed for a crystal phase is clearly visible as shown in Fig. 5(a). Such micro area analysis on the atomic scale will enable future observation of the changes in microstructure in the battery operation process, and hence is considered capable of playing a decisive role in the studies on degradation and performance improvement for all solid-state sulfide electrolytes. The SAD pattern corresponding to Fig. 5(a) also shows that the glass-ceramic samples clearly have a unique crystallinity. The strong diffraction ring displayed on the innermost side of SAD pattern has the interplanar distance of $6.02 \AA$ in agreement with the interplanar distance for $(01 \overline{2})$ of $\mathrm{Li}_{7} \mathrm{P}_{3} \mathrm{~S}_{11}$ (See Table 1). Interplanar distances for the remaining diffraction pattern are $3.46,3.02,2.84$, and $2.76 \AA$ in agreement with the interplanar distances of $(022),(2 \overline{1} \overline{1})$, $(1 \overline{3} \overline{3})$, and (103), respectively, for $\mathrm{Li}_{7} \mathrm{P}_{3} \mathrm{~S}_{11}$. As a reference, the diffraction ring displayed between $6.02 \AA$ and $3.46 \AA$ observed in the diffraction pattern was produced due to

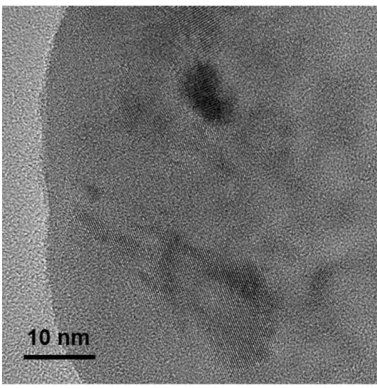

(a)

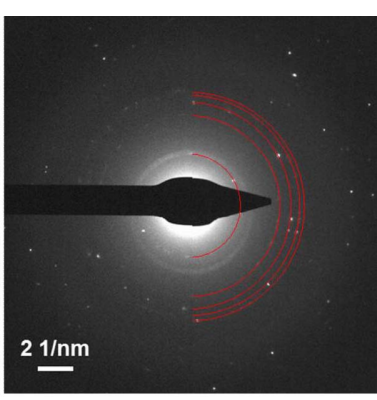

(b)
Fig. 5. (a) Bright field TEM image and (b) SAD pattern of an as-annealed LPS $(7: 3)$ particle with high lithiumion conducting crystalline structure. The SAD pattern is indexed as being from a $\mathrm{Li}_{7} \mathrm{P}_{3} \mathrm{~S}_{11}$ crystalline phase. 
Table 1. List of X-ray Diffraction Peaks for $\mathrm{Li}_{7} \mathrm{P}_{3} \mathrm{~S}_{11}$

\begin{tabular}{ccccc}
\hline$h$ & $k$ & $l$ & $d$ & $2 \boldsymbol{\theta}$ (deg.) \\
\hline 0 & 1 & -2 & 6.02989 & 14.679 \\
1 & -1 & 0 & 5.76091 & 15.368 \\
0 & 2 & -2 & 4.99369 & 17.747 \\
1 & -1 & 1 & 4.89023 & 18.126 \\
1 & 0 & -2 & 4.79967 & 18.471 \\
1 & -2 & 0 & 4.75500 & 18.646 \\
0 & 1 & -3 & 4.07140 & 21.812 \\
0 & 3 & 0 & 3.75172 & 23.696 \\
1 & 1 & 1 & 3.72498 & 23.869 \\
0 & 2 & 2 & 3.44140 & 25.869 \\
2 & -1 & -1 & 3.01344 & 29.621 \\
1 & 1 & -4 & 2.99218 & 29.836 \\
0 & 4 & -2 & 2.97929 & 29.968 \\
1 & -4 & 0 & 2.88933 & 30.924 \\
1 & -3 & 3 & 2.86352 & 31.210 \\
1 & 0 & 3 & 2.7784 & 33.192 \\
\hline
\end{tabular}

TEM aperture, and unrelated to the LPS $(7: 3)$ specimen. Based on this observation, it can be affirmed that the glassceramic powder of LPS $(7: 3)$ obtained through heat treatment clearly has a crystal structure of the high Li-ion conducting phase of $\mathrm{Li}_{7} \mathrm{P}_{3} \mathrm{~S}_{11}$ as confirmed in XRD.

Figure 6 shows the result of EDS mapping for the LPS $(7: 3)$ after heat treatment. As shown in Fig. 6, no major variations in contrast for distribution of the elements $\mathrm{P}$ and $\mathrm{S}$ are observed across the whole region of the sample. Individual elements are also distributed homogeneously in a uniform composition ratio after heat treatment. After the
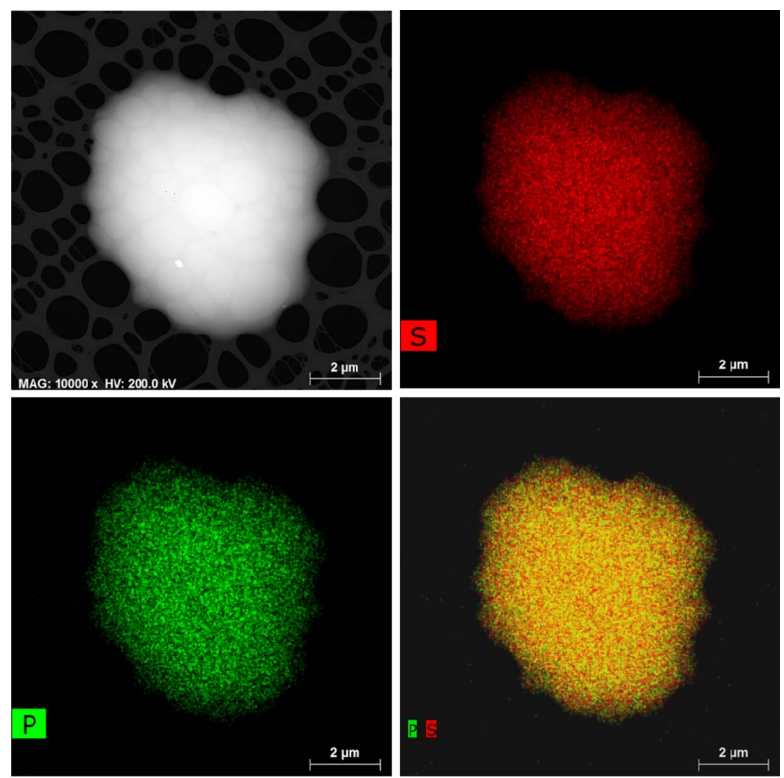

Fig. 6. TEM and EDS elemental mapping images of as-annealed LPS (7:3) particle. The green and red areas correspond to the elements of $\mathrm{P}$ and $\mathrm{S}$, respectively. heat treatment, the composition of LPS $(7: 3)$ matrix as confirmed through EDS scan of local areas was affirmed to be about 24 at.\% for S and about 76 at.\% for P. Therefore, the transition from the amorphous $\mathrm{Li}_{7} \mathrm{P}_{3} \mathrm{~S}_{11} \mathrm{P}$ to the crystalline $\mathrm{Li}_{7} \mathrm{P}_{3} \mathrm{~S}_{11}$ can be seen to have occurred without a large loss in $\mathrm{P}$ and S. Information on glass-ceramic crystal structures which used to be observed in bulk analysis such as XRD was satisfactorily affirmed in microscopic analysis area, and it was confirmed that the crystal structure of the highly conductive phase of $\mathrm{Li}_{7} \mathrm{P}_{3} \mathrm{~S}_{11}$, in particular was formed with the foundation placed on glass matrix through homogeneous chemical mixing throughout before and after heat treatment.

\section{Conclusions}

Advanced analysis based on TEM as a high-resolution microscopic analysis technique was conducted for the sulfide solid electrolyte materials, which used to depend on wide area-bulk analysis techniques such as XRD, NMR, and Raman due to the vulnerability to electron beam and ion beam despite the wide use as an electrolyte for all-solidstate batteries. High Li-ion conducting sulfide material of LPS (7:3) was synthesized into amorphous and glassceramic structures, respectively, by using high-energy milling equipment and follow-up heat treatment process. According to the XRD results together with the analysis results of TEM-SAD pattern and TEM bright-field image, the LPS $(7: 3)$ before heat treatment could be affirmed to be composed of amorphous $\mathrm{Li}_{7} \mathrm{P}_{3} \mathrm{~S}_{11}$. By using TEM-EDS, the amorphous $\operatorname{Li}_{7} \mathrm{P}_{3} \mathrm{~S}_{11}$ was shown to have about 78 and 22 at.\% of $\mathrm{S}$ and $\mathrm{P}$, respectively, as a matrix of a homogeneous composition distribution. After heat treatment at $260^{\circ} \mathrm{C}$ for $2 \mathrm{~h}$, the crystal planes could be affirmed in the high-magnification bright-field image of LPS $(7: 3)$. Also, the diffraction pattern displayed in the $\mathrm{SAD}$ pattern agreed with that of $\mathrm{Li}_{7} \mathrm{P}_{3} \mathrm{~S}_{11}$, allowing confirmation of the transition from the amorphous $\mathrm{Li}_{7} \mathrm{P}_{3} \mathrm{~S}_{11}$ to a crystalline phase on the homogeneous glass. Thus, the results of microscopic analysis on the atomic scale along with crystallographic understanding of sulfide solid electrolyte for all-solid-state batteries based on such advanced TEM techniques are expected to be able to play a decisive role in the future studies on performance and degradation improvement for the solid electrolytes.

\section{Acknowledgments}

This work was supported by the Energy Efficiency \& Resources Core Technology Program of the Korea Institute of Energy Technology Evaluation and Planning (KETEP) granted financial resource from the Ministry of Trade, Industry \& Energy, Republic of Korea (No. 2015202010 6100). 


\section{REFERENCES}

1. B. B. Owens and P. M. Skarstad, "Ambient Temperature Solid State Batteries," Solid State Ion., 53-56 665-72 (1992).

2. J. B. Goodenough and Y. Kim, "Challenges for Rechargeable Li Batteries," Chem. Mater., 22 [3] 587-603 (2010).

3. K. Takada, "Progress and Prospective of Solid-State Lithium Batteries," Acta Mater., 61 [3] 759-70 (2013).

4. J. G. Kim, B. Son, S. Mukherjee, N. Schuppert, A. Bates, O. Kwon, M. J. Choi, H. Y. Chung, and S. Park, "A Review of Lithium and Non-Lithium Based Solid State Batteries," J. Power Sources, 282 299-322 (2015).

5. M. Tatsumisago and A. Hayashi, "Sulfide Glass-Ceramic Electrolytes for All-Solid-State Lithium and Sodium Batteries," Int. J. Appl. Glass Sci., 5 [3] 226-35 (2014).

6. M. Tachez, J. P. Malugani, R. Mercier, and G. Robert, "Ionic Conductivity and Phase Transition in Lithium Thiophosphate $\mathrm{Li}_{3} \mathrm{PS}_{4}$," Solid State Ion., 14 181-85 (1984).

7. K. Takada, N. Aotani, K. Iwamoto, and S. Kondo, "Solid State Lithium Battery with Oxysulfide Glass," Solid State Ion., 86-88 877-82 (1996).

8. A. Hayashi, S. Hama, H. Morimoto, M. Tatsumisago, and T. Minami, "Preparation of $\mathrm{Li}_{2} \mathrm{~S}-\mathrm{P}_{2} \mathrm{~S}_{5}$ Amorphous Solid Electrolytes by Mechanical Milling," J. Am. Ceram. Soc., 84 [2] 477-79 (2001).

9. F. Mizuno, A. Hayashi, K. Tadanaga, and M. Tatsumisago, "New, Highly Ion-Conductive Crystals Precipitated from $\mathrm{Li}_{2} \mathrm{~S}-\mathrm{P}_{2} \mathrm{~S}_{5}$ Glasses," Adv. Mater., 17 [7] 918-21 (2005).

10. N. Kamaya, K. Homma, Y. Yamakawa, M. Hirayama, R.
Kanno, M. Yonemura, T. Kamiyama, Y. Kato, S. Hama, K. Kawamoto, and A. Mitsui, "A Lithium Superionic Conductor," Nat. Mater., 10 682-86 (2011).

11. G. Sahu, Z. Lin, J. Li, Z. Liu, N. Dudney, and C. Liang, "Air-Stable, High-Conduction Solid Electrolytes of ArsenicSubstituted $\mathrm{Li}_{4} \mathrm{SnS}_{4}$," Energy Environ. Sci., 7 1053-58 (2014).

12. D. Qian, C. Ma, K. L. More, Y. S. Meng, and M. Chi, "Advanced Analytical Electron Microscopy for Lithium-Ion Batteries," NPG Asia Mater., 7 e193 (2015).

13. A. Sakuda, A. Hayashi, and M. Tatsumisago, "Interfacial Observation between $\mathrm{LiCoO}$ Electrode and $\mathrm{Li}_{2} \mathrm{~S}-\mathrm{P}_{2} \mathrm{~S}_{5}$ Solid Electrolytes of All-Solid-State Lithium Secondary Batteries Using Transmission Electron Microscopy," Chem. Mater., 22 [3] 949-56 (2010).

14. J. H. Woo, J. E. Trevey, A. S. Cavanagh, Y. S. Choi, S. C. Kim, S. M. George, K. H. Oh, and S. H. Lee, "Nanoscale Interface Modification of $\mathrm{LiCoO}_{2}$ by $\mathrm{Al}_{2} \mathrm{O}_{3}$ Atomic Layer Deposition for Solid-State Li Batteries," J. Electrochem. Soc., 159 [7] A1120-24 (2012).

15. M. H. Hebb, "Electrical Conductivity of Siliver Sulfide," J. Chem. Phys., 20 [1] 185-190 (1952).

16. H. Yamane, M. Shibata, Y. Shimane, T. Junke, Y. Seino, S. Adams, K. Minami, A. Hayashi, and M. Tatsumisago, "Crystal Structure of a Superionic Conductor, $\mathrm{Li}_{7} \mathrm{P}_{3} \mathrm{~S}_{11}$ ", Solid State Ion., 178 1163-67 (2007).

17. M. Tatsumisago, M. Nagao, and A. Hayashi, "Recent Development of Sulfide Solid Electrolytes and Interfacial Modification for All-Solid-State Rechargeable Lithium Batteries," J. Asian Ceram. Soc., 1 [1] 17-25 (2013). 